\title{
Quantitative Measurement of $\gamma$-Ray and e-Beam Effects on Fiber Rayleigh Scattering Coefficient
}

\author{
Yongxiang $\mathrm{CHEN}^{1}$, Jiaqi $\mathrm{LI}^{2}$, Zinan WANG ${ }^{1 *}$, Andrei STANCĂLIE ${ }^{3}$, \\ Daniel IGHIGEANU ${ }^{3}$, Daniel NEGUT ${ }^{4}$, Dan SPOREA ${ }^{3}$, and Gangding PENG ${ }^{5}$ \\ ${ }^{1}$ Key Lab of Optical Fiber Sensing \& Communications, University of Electronic Science \&Technology of China, \\ Chengdu 611731, China \\ ${ }^{2}$ Metrology and Testing Center, China Academy of Engineering Physics (CAEP), Mianyang 621900, China \\ ${ }^{3}$ National Institute for Laser, Plasma and Radiation Physics Center for Advanced Laser Technologies, Magurele \\ RO-077125, Romania \\ 4 “Horia Hulubei” National Institute of Physics and Nuclear Engineering, Măgurele RO-077125, Romania \\ ${ }^{5}$ Photonics and Optical Communications, The University of New South Wales (UNSW), Sydney 2052, Australia \\ *Corresponding author: Zinan WANG_E-mail: znwang@uestc.edu.cn
}

\begin{abstract}
The effects of gamma ray ( $\gamma$-ray) radiation and electron beam (e-beam) radiation on Rayleigh scattering coefficient in single-mode fiber are experimentally investigated. Utilizing an optical time domain reflectometry (OTDR), the power distribution curves of the irradiated fibers are obtained to retrieve the corresponding radiation-induced attenuation (RIA). Based on the backscattering power levels and the measured RIAs, the Rayleigh scattering coefficients can be characterized quantitatively for each fiber sample. Under the given radiation conditions, Rayleigh scattering coefficients have been changed very little while RIAs have been changed significantly. Furthermore, simulations have been implemented to verify the validity of the measured Rayleigh scattering coefficient, including the splicing points.
\end{abstract}

Keywords: Gamma ray; electron beam; Rayleigh scattering; radiation-induced attenuation; Rayleigh scattering coefficient

Citation: Yongxiang CHEN, Jiaqi LI, Zinan WANG, Andrei STANCĂLIE, Daniel IGHIGEANU, Daniel NEGUȚ, et al., "Quantitative Measurement of $\gamma$-Ray and e-Beam Effects on Fiber Rayleigh Scattering Coefficient," Photonic Sensors, 2021, 11(3): 298 -304.

\section{Introduction}

Rayleigh scattering is an inherent scattering phenomenon of optical lightwave propagating through a medium. Scattered light is generated in various directions when light transmits in disordered medium with the random fluctuation of refractive index, due to its density fluctuation. Rayleigh systematically studied and explained the phenomenon of the blue sky [1] and further derived the $\lambda^{-4}$ law [2] from the elastic-solid and electromagnetic theory separately. Rayleigh scattering coefficient as a fundamental and important parameter of optical fiber, is of great significance in many applications [3-12], such as random fiber laser (RFL) [3-6], distributed fiber sensing like optical time domain reflectometry (OTDR) [7, 8], and optical frequency domain

Received: 05 December 2019 / Revised: 15 January 2020

(C) The Author(s) 2020. This article is published with open access at Springerlink.com

DOI: $10.1007 / \mathrm{s} 13320-020-0580-7$

Article type: Regular 
reflectometry (OFDR) $[9,10]$. Importantly, in these optical fiber applications, different fibers with different properties will bring completely different performances. Therefore, it is necessary to figure out the specific properties of the used optical fibers.

It is well known that irradiation could significantly alter fiber properties, and a great number of studies on the radiation-induced attenuation (RIA) in optical fibers were performed [13-17]. Besides, some reports described the mechanisms inducing microscopic and macroscopic changes under irradiation, containing compaction, radiation-induced emission, and RIA [18, 19]. In addition, some researches have studied the performance of irradiated fiber in the OFDR system $[20,21]$. However, these literatures didn't quantitatively reveal whether the Rayleigh scattering coefficient of the irradiated fiber has been changed or not, because the principle of such OFDR measurements is as follows: the refractive index of fiber will be altered by temperature/strain, causing the variation of the optical path length, and such length variation can be obtained by OFDR, using the Rayleigh backscattering signal of the probing lightwave. It should be noted that, Rayleigh backscattering coefficient is irrelevant to the temperature/strain resolution of OFDR (or frequency shift per temperature/strain unit).

In this paper, quantitative measurements of the effects of $\gamma$-ray and e-beam on Rayleigh scattering coefficients of optical fibers were carried out. The fibers used in this study are from a same reel of Corning (SMF-28e+) fiber. An OTDR system was employed to test the power distribution along the irradiated fibers and their corresponding RIAs were retrieved through data fitting; then Rayleigh scattering coefficients of these sample fibers were retrieved based on the measured backscattering power, and they almost remained constant with or without the radiations. This is the first report that the specific Rayleigh scattering coefficients of $\gamma$-ray and e-beam irradiated fibers have been measured in detail.

\section{Preparation of irradiated fiber samples}

$\gamma$-ray irradiation was performed at the Indian designed ${ }^{60} \mathrm{Co}$ GC-5000 irradiator. For $\gamma$-ray exposure, the samples were located in the middle of the cylindrical irradiation chamber (volume of $5000 \mathrm{~cm}^{3}$ ), at a distance of $10 \mathrm{~cm}$ from the base. The average temperature during the exposure was about $36^{\circ} \mathrm{C}$, and the irradiation was run at a dose rate of $1.2 \mathrm{~Gy} / \mathrm{s}$. The dose rate and the total dose were measured with $3.3 \%$ standard deviation. The dosimetry used in the system was the type of ethanol-chlorobenzene (ECB) with oscillometric readout, traceable at the National Physical Laboratory, by RISOE HDRL.

Some optical fiber samples were exposed to e-beam radiation at the "traveling-wave" linear accelerator which belongs to the Institute for Laser, Plasma and Radiation Physics (pulse duration of $4 \mu \mathrm{s}$, operating current of $3.5 \mu \mathrm{A}-5.0 \mu \mathrm{A}$, and frequency of $50 \mathrm{~Hz}$, and electron energy in the high power regime of $5.5 \mathrm{MeV}$ ). The employed dose rate was measured to be $26.7 \mathrm{~Gy} / \mathrm{s}$. The fibers were placed at $60 \mathrm{~cm}$ from the accelerator exit window. For the dosimetry, a graphite calorimeter, calibrated at Risø High Dose Reference Laboratory, was used.

Six pieces of common single-mode fibers were irradiated with $\gamma$-ray (represented by $G 1, G 2$, and G3, corresponding to $2 \mathrm{kGy}, 12 \mathrm{kGy}$, and $36 \mathrm{kGy}$ ) and e-beam (represented by E1,E2, and E3, corresponding to $5 \mathrm{kGy}, 25 \mathrm{kGy}$ and $75 \mathrm{kGy}$ ) respectively, each of them with a length of $100 \mathrm{~m}$. Besides, an additional $100 \mathrm{~m}$ identical SMF (SMF2) from the same reel fiber was utilized as a reference sample fiber without exposing it to any radiation. All of these seven sample fibers were wound into fiber coils with a diameter of $8 \mathrm{~cm}$.

\section{Measurement setups}

\subsection{OTDR curves acquisition}

In order to measure the RIAs of the irradiated fiber samples, a setup was built based on an OTDR 
manufactured by EXFO, with $5 \mathrm{~ns}$ pulse width (corresponding to $0.5 \mathrm{~m}$ spatial resolution), $1550 \mathrm{~nm}$ working wavelength, and $180 \mathrm{~s}$ average time. The sketch of the test system is shown in Fig. 1. A piece of $520 \mathrm{~m} \mathrm{SMF}$ (SMF1) wound around a $15 \mathrm{~cm}$ diameter coil was placed between the OTDR and SMF2 which was a reference to make sure fiber samples were properly wound without extra bending loss. The fiber under test (FUT), irradiated sample fiber, was connected to the SMF2 and tested one by one. In order to form an anti-reflection termination, the far end of the FUT was fused with an $8 \%$ oblique-angled pigtail and immersed into index matching liquid. The refractive index of the index matching liquid was 1.47 .

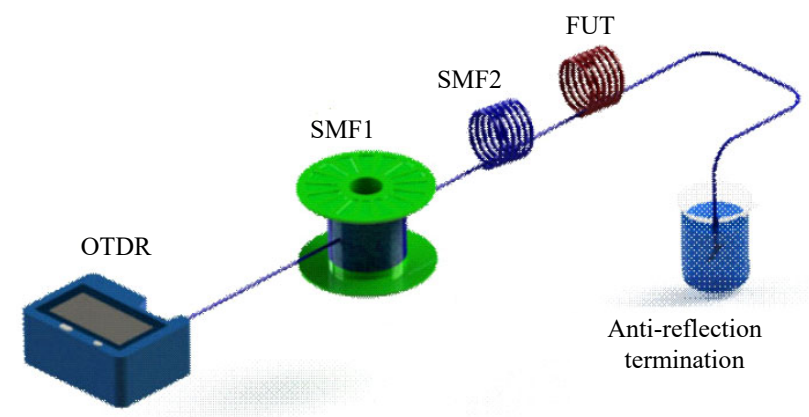

Fig. 1 OTDR-based attenuation measurement experimental setup.

\subsection{Backscattering integral power measurement}

Figure 2 depicts the measurement system for backscattering integral power from sample fibers. An amplified spontaneous emission (ASE) source was used as the light source and connected to the $1 \mathrm{st}$ port of the fiber optical circulator. The wavelength span of the ASE was from $1520 \mathrm{~nm}$ to $1570 \mathrm{~nm}$, and $10 \mathrm{~dB}$ bandwidth was $35.2 \mathrm{~nm}$. The $9.86 \mathrm{dBm}$ input lightwave transmitted through the 2 nd port of the circulator into FUT. The backward Rayleigh scattering lightwave was output from the 3rd port of circulator eventually and detected by an optical power meter (PM). Similarly, the far terminations of sample fibers were processed with an $8 \%$ oblique-angled pigtail and immersed into index matching liquid to eliminate the effects of Fresnel reflections in the process of measuring backscattering power. By the way, the two ports of the FUT and the 2nd port of circulator were directly fused together instead of being connected by a flange, so there was no need to worry about any spurious back reflection coming from the circulator. Importantly, the PM did not have any readout (the minimum detected power is $-50 \mathrm{dBm}$ ) when there was no FUT, which meant that the cross talk of the circulator between the 1 st port and 3rd port (less than $-60 \mathrm{dBm}$ ) was too small to have any influence.

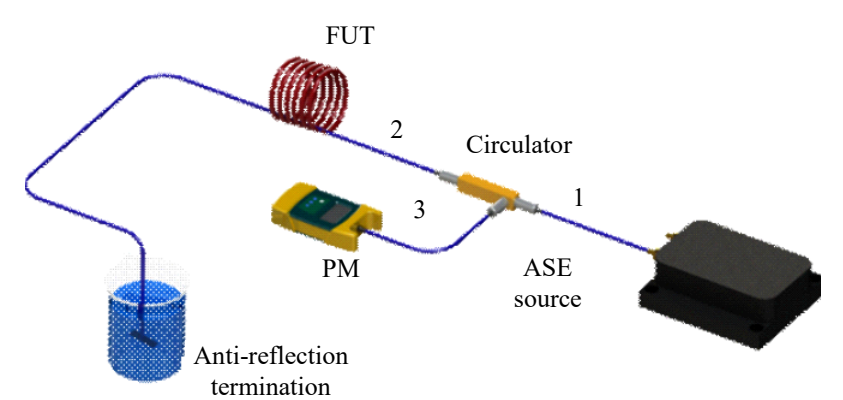

Fig. 2 Backscattering integral optical power measurement setup.

\section{Experimental results}

\subsection{Effects of $\gamma$-ray and e-beam radiation on fiber loss}

The optical power distributions along fiber in sample fibers can be directly obtained using the setup in Fig. 1 and the results are shown in Figs.3(a) and 3(b), respectively for the fibers exposed to $\gamma$-ray and e-beam radiation. Apparently, the optical power distribution in $100 \mathrm{~m}$ SMF2 (which had a typical loss of $0.18 \mathrm{~dB} / \mathrm{km}$ ) was highly consistent with the one in $520 \mathrm{~m} \mathrm{SMF1,} \mathrm{which} \mathrm{meant} \mathrm{that} \mathrm{they} \mathrm{had} \mathrm{the}$ same Rayleigh scattering coefficient. It also suggested that the winding method of the sample fibers did not affect the fiber loss. Moreover, the optical power curves of the irradiated fibers did not have sharp increase and decrease, which meant that the attenuation was relatively uniform along the fiber. These specific slopes were obtained through fitting the data of power distributions in the least-squares sense, as indicated in the regions 
labeled FUT of Figs. 3(a) and 3(b), and the calculated RIA coefficients are shown in the insets. In addition, for the sample fibers with anti-reflection, a conspicuous drop can be observed at the end of the power distribution curves, indicating that there was no reflection, which was also significant for the measurement of Rayleigh scattering coefficient.

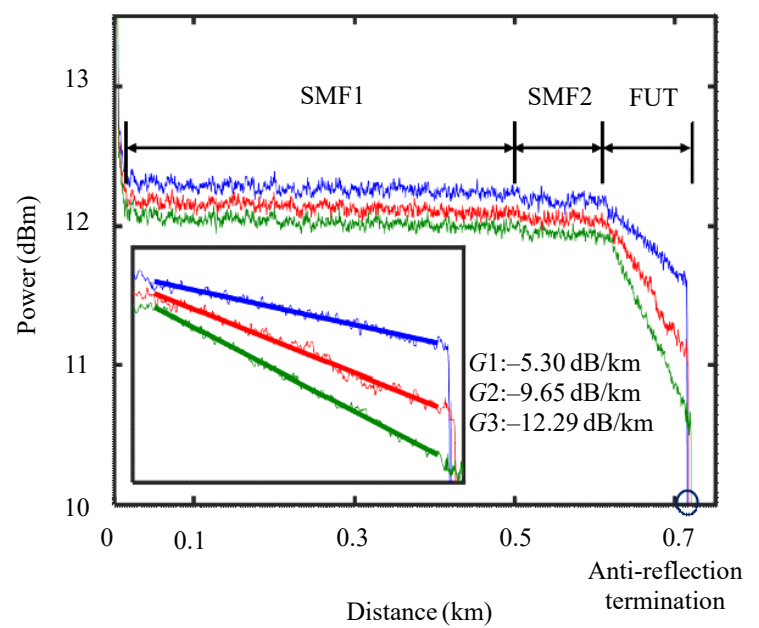

(a)

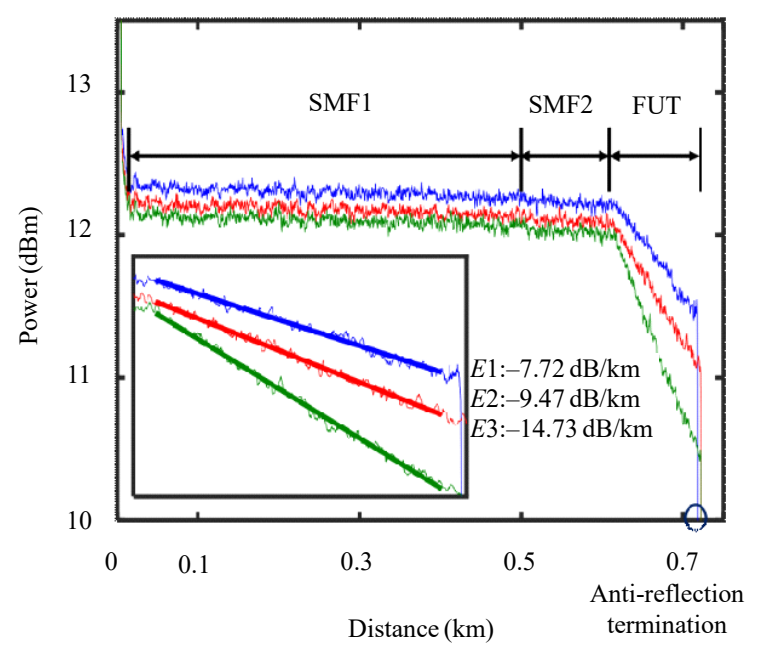

(b)

Fig. 3 Measured OTDR curves of the irradiated fibers: (a) $\gamma$-ray irradiated fibers and (b) e-beam irradiated fibers.

The trends of the RIA coefficients of the fiber are shown in Fig.4, where the black and red lines correspond to $\gamma$-ray radiation and e-beam radiation respectively. The results show that although both types of radiation enlarge fiber attenuation, the growth trends of different irradiation type are different. In the case of $\gamma$-ray radiation
( $2 \mathrm{kGy}-36 \mathrm{kGy}$ ), throughout the entire range, RIAs become larger as the irradiation increases, but the growth rate of $2 \mathrm{kGy}$ to $12 \mathrm{kGy}$ is greater than the growth rate of $12 \mathrm{kGy}$ to $36 \mathrm{kGy}$. This tendency highly coincides with the recent report [22]. In the range of e-beam radiation intensity $(5 \mathrm{kGy}-75 \mathrm{kGy})$, the RIA coefficients are approximately linear with the radiation intensity, which means that different irradiations have different mechanisms for changing fiber loss. As a result, such different changes could be used in different applications. For example, compared with the $\gamma$-ray irradiated fiber, the e-beam irradiated fiber could use its RIA to detect the dose of irradiation.

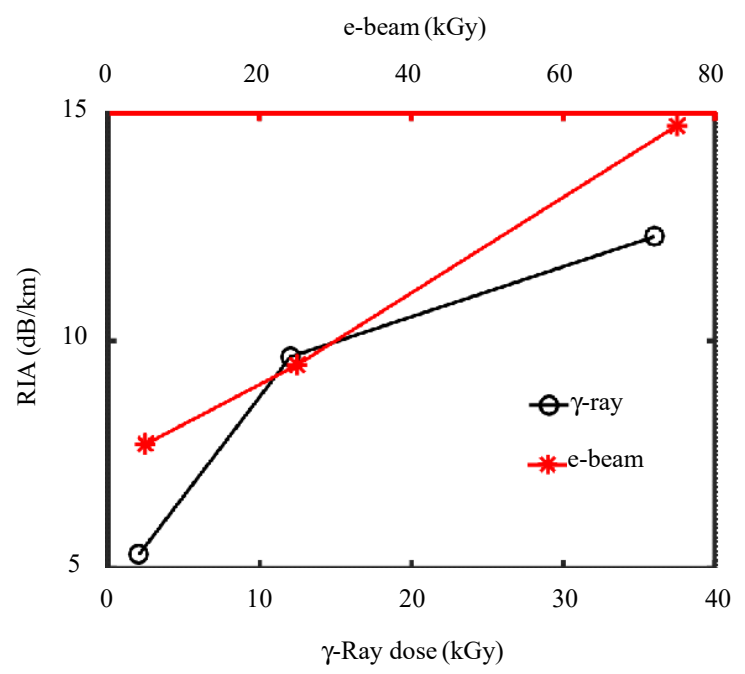

Fig. 4 RIA coefficients curves for the sample fibers exposed to $\gamma$-ray radiation (black line) and e-beam radiation (red line).

\subsection{Effects of $\gamma$-ray and e-beam radiation on Rayleigh scattering coefficient}

In fact, the obtained backscattering integral power represents the contributions of the attenuation and the Rayleigh scattering effects as the fiber tails are specially processed without reflection at the far ends. Besides, the injected light power is too small to bring any nonlinear effects. Therefore, at the back receiving module, the received Rayleigh backward scattering component is an integral result along fiber, with the absorption attenuation evenly distributing along the fiber, the received backscattering power from the 3rd port of circulator can be expressed as [23] 


$$
P_{\mathrm{bs}}=P_{\text {in }} \cdot \int_{0}^{L} \varepsilon_{s} \cdot \mathrm{e}^{-2 \alpha_{s} l} \mathrm{~d} l
$$

where $s=G 1, \cdots, G 3, E 1, \cdots, E 3$ denotes the labels of exposed sample fibers, $\alpha_{s}$ represents the optical loss coefficient in sample fibers, and $L$ is the length of the sample fiber. $P_{\text {in }}$ and $P_{\mathrm{bs}}$ express the injected optical power and the backscattering power, respectively. The reflectivity of a small section $\mathrm{d} l$ in the fiber is represented by the integral term $P_{\text {in }} \cdot \mathrm{e}^{-2 \alpha_{s} l} \cdot \varepsilon_{s} \cdot \mathrm{d} l$, in which $\varepsilon_{s}$ is the Rayleigh scattering factor.

Table 1 Measured and calculated parameters.

\begin{tabular}{ccccc}
\hline $\begin{array}{c}\text { Fiber } \\
\text { sample }\end{array}$ & $\begin{array}{c}\text { Radiation dose } \\
(\mathrm{kGy})\end{array}$ & $\alpha_{\mathrm{s}}(\mathrm{dB} / \mathrm{km})$ & $\begin{array}{c}P_{\mathrm{bs}} \\
(\mathrm{dBm})\end{array}$ & $\begin{array}{c}\mathcal{E}_{s} \\
\left(10^{-8} \cdot \mathrm{m}^{-1}\right)\end{array}$ \\
\hline SMF2 & 0 & 0.18 & -44.2 & 5.63 \\
$G 1$ & 2 & 5.30 & -44.7 & 5.64 \\
$G 2$ & 12 & 9.65 & -45.2 & 5.52 \\
$G 3$ & 36 & 12.29 & -45.3 & 5.70 \\
$E 1$ & 5 & 7.72 & -45.0 & 5.55 \\
$E 2$ & 25 & 9.47 & -45.0 & 5.76 \\
$E 3$ & 75 & 14.73 & -45.5 & 5.73 \\
\hline
\end{tabular}

Based on the setup in Fig. 2, the backward scattering power was measured at the input laser power $P_{\text {in }}$ of $9.86 \mathrm{dBm}$. Then, through (1), the Rayleigh scattering coefficients are calculated as shown in Table 1. Obviously, compared with SMF2, the result suggests that the Rayleigh scattering coefficients of the fibers exposed to both $\gamma$-ray and e-beam radiations do not show any obvious enhancement or decline. The irradiated fibers' and the SMF2's Rayleigh scattering coefficients are concentrated in the range of $5.5 \times 10^{-8} \mathrm{~m}^{-1}$ to $5.8 \times 10^{-8} \mathrm{~m}^{-1}$. Ignoring these small differences (less than $2 \%$ ), it can be considered that both the $\gamma$-ray and e-beam irradiations do not obviously change the Rayleigh scattering coefficient of the fiber. The differences (less than $2 \%$ ) between the measured Rayleigh scattering coefficients of 6 fibers are small, and such differences come from the $0.01 \mathrm{~dB}$ uncertainty of the power meter readout, as well as the fluctuation of the Rayleigh scattering coefficient along the fiber reel, from which the 6 fiber samples were obtained.

\subsection{Re-examination of the acquired Rayleigh scattering coefficients and RIAs}

For the purpose of verifying the acquired Rayleigh scattering coefficients and RIAs about the irradiated fibers (especially its splicing point with SMF), some simulations have been done with the parameters in Table 1, as shown in Fig. 5. The simulation model is a common OTDR simulation model, and the scattered light at point $Z$ is expressed as

$$
P_{\mathrm{bs}}(Z)=P_{\mathrm{in}} \cdot \sum_{l=Z}^{Z+W / 2} \varepsilon_{s}(l) \cdot \mathrm{e}^{-2 \alpha_{s} l}
$$

where $Z$ represents the distance between the starting point and the point $Z$. $P_{\text {in }}$ represents the intensity of the starting point, $W$ means the length of pulse width, and $\varepsilon_{s}(l)$ shows the Rayleigh scattering coefficient at point $l$. $\alpha_{s}$ corresponds to the attenuation, which is obtained from the slope of the OTDR curve.

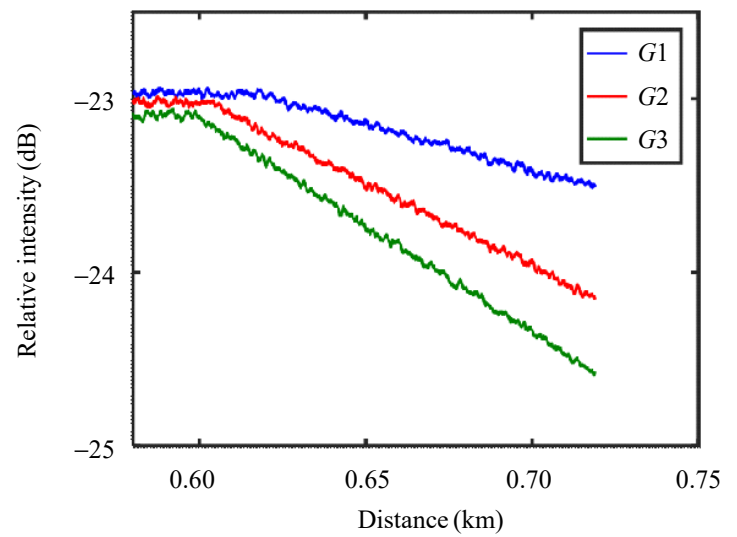

(a)

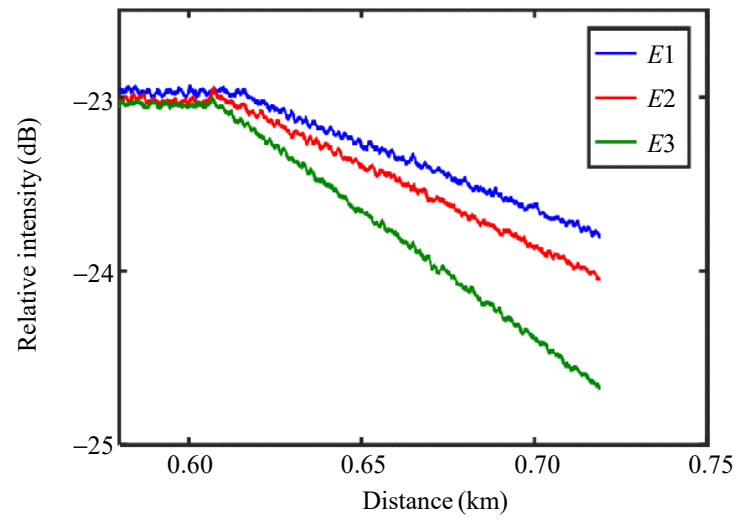

(b)

Fig. 5 Simulated OTDR curves of the (a) $\gamma$-ray and (b) e-beam irradiated fibers. 
In the simulation model, for each fiber sample, the Rayleigh scattering coefficient is preset to be Rayleigh distribution along the fiber [24], with a certain mean value and $0.15 \times 10^{-8}$ variance. The mean of the Rayleigh scattering coefficients of different samples are the last column of Table1. In order to compare with the experimental data, the 620-meter SMF spliced with 100-meter FUT was simulated. Besides, the simulation model also considers the pulse width of the input light, which makes the results more accurate. The simulation results are consistent with the experimental results [Figs. 3 (a) and (b)], including the data at the locations of the SMF and FUT splicing point, further demonstrating that the measurements are correct and two irradiation schemes do not significantly alter Rayleigh scattering coefficient of the fiber.

\section{Conclusions}

In conclusion, the effects on Rayleigh scattering coefficient in standard SMF exposed to $\gamma$-ray and e-beam radiation were studied in detail. Firstly, the power distributions along the irradiated fibers were measured with an OTDR for the corresponding RIA. Then, based on the backward power measurement and the obtained RIAs, Rayleigh scattering coefficients were characterized quantitatively. Simulations have been implemented to verify the validity of retrieved RIAs and Rayleigh scattering coefficients, especially at the splicing points. The results show that RIA increases as the radiation dose increases for both $\gamma$-ray and e-beam radiations with different trends, while the Rayleigh scattering coefficients almost remain the same.

\section{Acknowledgment}

This work was supported by the National Natural Science Foundation of China (Grant Nos. 41527805 and 61635005), Sichuan Youth Science and Technology Foundation (Grant No. 2016JQ0034), and the 111 Project (Grant No. B14039). The Romanian authors acknowledge the support of the Romanian Executive Agency for Higher Education, Research, Development and Innovation Funding (Grant No. UEFISCDI), under the contract "Sensor Systems for Secure Operation of Critical Installations". G. Peng acknowledges the support by Science and Technology Commission of Shanghai Municipality, China (Grant Nos. SKLSFO2015-01 and 15220721500) and by the Hisilicon Innovation Research Program (HIRP) (Grant No. HO2017050001CZ).

Open Access This article is distributed under the terms of the Creative Commons Attribution 4.0 International License (http://creativecommons.org/licenses/by/4.0/), which permits unrestricted use, distribution, and reproduction in any medium, provided you give appropriate credit to the original author(s) and the source, provide a link to the Creative Commons license, and indicate if changes were made.

\section{References}

[1] A. T. Young, "Rayleigh scattering," Applied Optics, 1981, 20(4): 533-535.

[2] L. Rayleigh, "XXXIV. On the transmission of light through an atmosphere containing small particles in suspension, and on the origin of the blue of the sky," The London, Edinburgh, and Dublin Philosophical Magazine and Journal of Science, 1899, 47(287): 375-384.

[3] S. K. Turitsyn, S. A. Babin, A. E. El-Taher, P. Harper, D. V. Churkin, S. I. Kablukov, et al., "Random distributed feedback fibre laser," Nature Photonics, 2010, 4(4): 231.

[4] Z. N. Wang, H. Wu, M. Fan, L. Zhang, Y. Rao, W. Zhang, et al., "High power random fiber laser with short cavity length: theoretical and experimental investigations," IEEE Journal of Selected Topics in Quantum Electronics, 2014, 21(1): 10-15.

[5] D. V. Churkin, S. A. Babin, A. E. El-Taher, P. Harper, S. I. Kablukov, V. Karalekas, et al., "Raman fiber lasers with a random distributed feedback based on Rayleigh scattering," Physical Review A, 2010, 82(3): 033828.

[6] X. Wang, D. Chen, H. Li, L. She, and Q. Wu, "Random fiber laser based on artificially controlled backscattering fibers," Applied Optics, 2018, 57(2): 258-262.

[7] Z. N. Wang, L. Zhang, S. Wang, N. T. Xue, F. Peng, M. Q. Fan, et al., "Coherent $\Phi$-OTDR based on I/Q demodulation and homodyne detection," Optics Express, 2016, 24(2): 853-858. 
[8] Z. N. Wang, B. Zhang, J. Xiong, Y. Fu, S. T. Lin, J. L. Jiang, et al., "Distributed acoustic sensing based on pulse-coding phase-sensitive OTDR," IEEE Internet of Things Journal, 2019, 6(4): 6117-6124.

[9] S. Wang, X. Fan, Q. Liu, and Z. He, "Distributed fiber-optic vibration sensing based on phase extraction from time-gated digital OFDR," Optics Express, 2015, 23(26): 33301-33309.

[10] S. Loranger, M. Gagné, V. Lambin-Iezzi, and R. Kashyap, "Rayleigh scatter based order of magnitude increase in distributed temperature and strain sensing by simple UV exposure of optical fibre," Scientific Reports, 2015, 5: 11177.

[11] A. Yan, S. Huang, S. Li, R. Chen, P. Ohodnicki, M. Buric, et al., "Distributed optical fiber sensors with ultrafast laser enhanced Rayleigh backscattering profiles for real-time monitoring of solid oxide fuel cell operations," Scientific Reports, 2017, 7(1): 9360.

[12] A. Hartog, "A distributed temperature sensor based on liquid-core optical fibers," Journal of Lightwave Technology, 1983, 1(3): 498-509.

[13] D. Johlen, P. Knappe, H. Renner, and E. Brinkmeyer, "UV-induced absorption, scattering and transition losses in UV side-written fibers," OFC/IOOC., San Diego, CA, USA, Feb. 21, 1999, 3: 50-52.

[14] P. L. Mattem, L. M. Watkins, C. D. Skoog, J. R. Brandon, and E. H. Barsis, "The effects of radiation on the absorption and luminescence of fiber optic waveguides and materials," IEEE Transactions on Nuclear Science, 1974, 21(6): 81-95.

[15] E. J. Friebele, "Optical fiber waveguides in radiation environments," Optical Engineering, 1979, 18(6): 186552.

[16] M. Kyoto, Y. Chigusa, M. Ohe, H. Go, M. Watanabe, T. Matsubara, et al., "Gamma-ray radiation hardened properties of pure silica core single-mode fiber and its data link system in radioactive environments," Journal of Lightwave Technology, 1992, 10(3):
289-294.

[17] H. Henschel, O. Kohn, H. U. Schmidt, E. Bawirzanski, and A. Landers, "Optical fibres for high radiation dose environments," IEEE Transactions on Nuclear Science, 1994, 41(3): 510-516.

[18] W. Primak, "Fast-neutron-induced changes in quartz and vitreous silica," Physical Review, 1958, 110(6): 1240 .

[19] S. Girard, J. Kuhnhenn, A. Gusarov, B. Brichard, M. Van Uffelen, Y. Ouerdane, et al., "Radiation effects on silica-based optical fibers: Recent advances and future challenges," IEEE Transactions on Nuclear Science, 2013, 60(3): 2015-2036.

[20] S. Rizzolo, A. Boukenter, E. Marin, M. Cannas, J. Perisse, S. Bauer, et al., "Vulnerability of OFDR-based distributed sensors to high $\gamma$-ray doses," Optics Express, 2015, 23(15): 18997-19009.

[21] C. Sabatier, S. Rizzolo, A. Morana, T. Allanche, T. Robin, B. Cadier, et al., "6-mev electron exposure effects on OFDR-based distributed fiber-based sensors," IEEE Transactions on Nuclear Science, 2018, 65(8): 1598-1603.

[22] J. Wu, L. Ma, F. Tu, and Z. He, "Investigation of radiation effect on single-mode fiber for distributed radiation sensing application," in 2018 Asia Communications and Photonics Conference (ACP), Hangzhou, China, Oct. 26-29, 2018, pp. 1-3.

[23] Z. N. Wang, M. Q. Fan, L. Zhang, H. Wu, D. V. Churkin, Y. Li, et al., "Long-range and high-precision correlation optical time-domain reflectometry utilizing an all-fiber chaotic source," Optics Express, 2015, 23(12): 15514-15520.

[24] L. B. Liokumovich, N. A. Ushakov, O. I. Kotov, M. A. Bisyarin, and A. H. Hartog, "Fundamentals of optical fiber sensing schemes based on coherent optical time domain reflectometry: Signal model under static fiber conditions," Journal of Lightwave Technology, 2015, 33(17): 3660-3671. 